\title{
Synergistic and Competitive Effects between Zinc Dialkyldithiophosphates and Modern Generation of Additives in Engine Oil
}

\author{
Khai K. Huynh, Kiet A. Tieu *(D) and Sang T. Pham (D) \\ School of Mechanical, Materials, Mechatronics and Biomedical Engineering, University of Wollongong, \\ Wollongong, NSW 2500, Australia; kkh981@uow.edu.au (K.K.H.); tsp310@uowmail.edu.au (S.T.P.) \\ * Correspondence: ktieu@uow.edu.au
}

check for

updates

Citation: Huynh, K.K.; Tieu, K.A.; Pham, S.T. Synergistic and Competitive Effects between Zinc Dialkyldithiophosphates and Modern Generation of Additives in Engine Oil. Lubricants 2021, 9, 35. https:// doi.org/10.3390/lubricants9040035

Received: 13 February 2021

Accepted: 26 March 2021

Published: 31 March 2021

Publisher's Note: MDPI stays neutral with regard to jurisdictional claims in published maps and institutional affiliations.

Copyright: (c) 2021 by the authors. Licensee MDPI, Basel, Switzerland. This article is an open access article distributed under the terms and conditions of the Creative Commons Attribution (CC BY) license (https:// creativecommons.org/licenses/by/ $4.0 /)$.

\begin{abstract}
The increasing demand for low-viscosity engine oil has underscored the role of zinc dialkyldithiophosphates (ZDDP) as a conventional anti-wear and antioxidant additive. It is essential to investigate the influence of modern additives such as cyclopropanecarboxylic acid (CPCa) and Ni nanoparticles on the tribological performance of ZDDP for practical commercial oil application. According to the experimental results, Ni nanoparticles formed a protective film that exhibited a synergistic effect with ZDDP. A significantly higher concentration of sulphur in the tribofilm was detected compared to ZDDP by itself, which was responsible for a 27.6\% lower wear loss. Meanwhile, a competitive effect between CPCa and ZDDP resulted in a dramatic increase in friction and unstable anti-wear performance. This was demonstrated by a localized formation of the ZDDP tribofilm on the wear surfaces after the friction test. These results have highlighted the synergistic and competitive effects of emerging additives (CPCa and Ni nanoparticles) in the ZDDP tribofilm formation between the sliding steel contacts. This further suggests a new approach to increase the efficiency of ZDDP's tribological performance at cold start-up processes.
\end{abstract}

Keywords: ZDDP; Ni nanoparticles; carbon-based tribofilm; friction; wear

\section{Introduction}

In recent years, due to the increasing demand for low-viscosity engine oils [1], zinc dialkyldithiophosphate (ZDDP) as a conventional lubricating additive has been widely used. There is a need for ZDDP to reduce the friction and wear of moving parts to improve engine efficiency [2]. Its functional properties, as extreme pressure additive and antioxidant, have been widely recognized and employed in the transportation industry since the 1940s [3]. Under extreme pressure conditions, ZDDP decomposes and reacts with the oxide layer of the sliding substrate, forming the pad-like structure tribofilm. This tribolayer not only acts as a cushion preventing wear but also inhibits the oxidation process on the sliding surface $[4,5]$. Due to its strong absorption strength on steel substrate, the addition of this additive to the commercial engine oil may not be completely replaced in the near future [6]. However, the anti-friction performance of ZDDP film is controversial because of its rough film formation in the boundary lubrication regime [7,8]. In addition, the reduction of ZDDP usage is currently encouraged because it produces harmful emissions of $\mathrm{Zn}, \mathrm{P}$, and S products that can deteriorate the performance of the engine system's catalytic converter and induce negative effects to the environment [9]. As a result, recent research on engine oil additives has been looking for new additives or innovative solutions to limit the amount of ZDDP usage and/or improve its tribofilm anti-friction performance [10].

Among the approaches, carbon-based tribofilm has emerged as a new solution that can resolve the problems relating to the harmful wastes of conventional additives. An attempt to generate the carbon-based film with a structure analogous to diamond-like-carbon (DLC) coatings has received much interest because of their outstanding performance in 
friction and wear reductions in engineering systems [11]. Intriguingly, their tribological characteristics are dominantly dependent on the $\mathrm{sp}^{2}$ - and $\mathrm{sp}^{3}$ - portions of the carbon hybridization and the fraction of doped hydrogen [12]. There are several methods to grow these films on the sliding substrates including magnetron sputtering or using chemical vapor deposition technique. Recently, the use of cycloalkane compounds, which are easily dissociated and polymerized under stressed-shearing conditions, has become a hot research topic $[13,14]$. In particular, cyclopropanecarboxylic acid (CPCa), a carbonprecursor additive with a highly strained triangle ring and an acid carboxylic functional group, has recently received much attention due to its ability to generate in situ carbonbased tribofilm on the steel surfaces [13]. Additionally, this additive is famous for its spontaneous absorption to the steel surface due to the presence of the acid carboxylic group [15]. Although there have been many studies investigating the ZDDP film formation on DLC substrate [16,17], the influence of CPCa as an in situ carbon-based tribofilm formation additive on the ZDDP-derived tribofilm has not been studied yet.

Apart from carbon-based tribofilm, nanoparticles have been considered as another potential extreme pressure additive that enters the micro-roughness to polish the interfacing surfaces during the sliding process [18]. These materials have been widely used since the 1900s and comprise a variety of chemical compositions like metal oxide, carbon derivatives, metals, nanocomposites, etc. Four tribological mechanisms that allow "smooth" contact from nanoparticles in oil lubrication include rolling, protective film, mending, and polishing effects [19]. According to Spikes et al. [20], the main advantage of nanoparticles in friction reduction comes from the minor interaction with other engine oil additives in additive packages. In particular, metallic nanoparticles with unique chemical and physical characteristic are famous for the ability to mechanically form their protective tribofilms on various sliding substrates to improve the load-carrying capacity of the contact areas [18]. There have been many solid metallic nanoparticles that have been studied and employed such as iron [21,22], molybdenum [23], or palladium [24]. Among them, the nickel nanoparticle emerges as one of the softest nanoparticles with the lowest melting temperature which is more beneficial to promoting a compact protective film on the contacting area via the nanoparticles' sintering by flash temperature [25]. Nevertheless, the tribological property of this nanomaterial under boundary lubrication regime as well as the interaction between the Ni-rich film and ZDDP film has not been yet studied.

In practice, many additives have been proven to have synergistic effects on each other when they were combined in a lubricant package $[26,27]$. Nevertheless, there are also a lot of additive combinations which exhibit competitive effects and reduce the functional quality of the lubricant fluid $[28,29]$. Therefore, it is essential to investigate the effect of $\mathrm{CPCa}$ and Ni nanoparticles in the tribological performance of ZDDP-containing Polyalphaolefin (PAO) oil. This study aims to identify the synergistic and competitive effects of modern additives in the generation of the ZDDP tribofilm during sliding. All tribo-experiments will be carried at room temperature to simulate the engine's cold start-up condition where the friction is significantly high [30]. The significant findings in this study will advance the understanding of tribochemical reactions between additives and provide the opportunity for better engine oil package design in the future.

\section{Experimental Preparation}

\subsection{Lubricant Preparation}

In this work, PAO4 oil as the base lubricant was supplied by the Exxonmobile company. Meanwhile, other lubricant additives including solid Ni nanoparticles and liquid CPCa were purchased from Sixth Element Materials Technology Co. Ltd. (NSW, Australia) and Sigma-Aldrich Corporation (NSW, Australia), respectively. ZDDP as a conventional lubricating additive was provided by Luoyang Trunnano Tech Co., Ltd. (Luoyang, China). These additives were directly blended into the base lubricant without any modification. While the oil-soluble additives (e.g., CPCa and ZDDP) were blended in PAO4 by magnetic stirring, an ultrasonic bath was employed to uniformly disperse the Ni nanoparticles. In 
particular, the dispersing duration for the samples containing Ni nanoparticles was $8 \mathrm{~h}$. The total concentration of oil-soluble additives in PAO oil was $2.5 \mathrm{wt} \%$, while the Ni additive's concentration was $0.1 \mathrm{wt} \%$. To examine the competitive or synergistic effects between CPCa and ZDDP in terms of the tribofilm formation on the steel surfaces, the concentration of each additive in the oil was then reduced to $1.25 \mathrm{wt} \%$. This test concentration was chosen to examine the effect of different concentrations of CPCa and ZDDP on the oil mixture tribological performance. The detailed concentration of the experimental samples can be found in Table 1.

Table 1. Experimental denotation and concentration.

\begin{tabular}{|c|c|c|}
\hline Experimental Sample & Denoted & Concentration \\
\hline Pure PAO oil & PAO & $\mathrm{PAO}$ \\
\hline PAO oil containing ZDDP & ZDDP & $\mathrm{PAO}+2.5 w \mathrm{t} \% \mathrm{ZDDP}$ \\
\hline PAO oil containing $\mathrm{CPCa}$ & $\mathrm{CPCa}$ & $\mathrm{PAO}+2.5 w \mathrm{t} \% \mathrm{CPCa}$ \\
\hline $\begin{array}{l}\text { PAO oil containing } \\
\text { Ni nanoparticles }\end{array}$ & $\mathrm{Ni}$ & $\mathrm{PAO}+0.1 w \mathrm{t} \% \mathrm{Ni}$ \\
\hline $\begin{array}{l}\text { PAO oil containing ZDDP } \\
\text { and } \mathrm{CPCa}\end{array}$ & $\mathrm{ZDDP}+\mathrm{CPCa}$ & $\mathrm{PAO}+1.25 w \mathrm{t} \% \mathrm{ZDDP}+1.25 w \mathrm{t} \% \mathrm{CPCa}$ \\
\hline $\begin{array}{c}\text { PAO oil containing ZDDP and } \\
\text { Ni combination }\end{array}$ & $\mathrm{ZDDP}+\mathrm{Ni}$ & $\mathrm{PAO}+2.5 w \mathrm{t} \% \mathrm{ZDDP}+0.1 w \mathrm{t} \% \mathrm{Ni}$ \\
\hline
\end{tabular}

\subsection{Testing Methods}

The tribotests were carried out using a Bruker UMT Tribolab machine (Billerica, MA, USA) at $25{ }^{\circ} \mathrm{C}$. The GCr15 (AISI52100 grade) steel ball with $6.35 \mathrm{~mm}$ diameter and $0.02 \mu \mathrm{m}$ surface roughness was selected as the upper stationary specimen, while the lower rotating specimen was the AISI52100 bearing steel disc with $30 \mathrm{~mm}$ diameter, $3 \mathrm{~mm}$ thickness, and $0.578 \pm 0.088 \mu \mathrm{m}$ average roughness. Before the test, acetone and then ethanol were used to ultrasonically clean the tribo-counterparts to remove any contaminants on the surfaces. The applied force of the ball-on-disc experiments was $10 \mathrm{~N}$ which corresponds to 1.26 GPa Hertzian pressure. The friction tests were conducted on the disc surface at different positions from the disc center with the remaining constant of the linear speed of $0.1 \mathrm{~m} / \mathrm{s}$ in $1 \mathrm{~h}$ which corresponded to the total sliding distance of $360 \mathrm{~m}$. Based on Dowson-Higginson equations [31], the lubricant film thickness for the chosen conditions was calculated to be $133 \mathrm{~nm}$ and the lambda value was 0.230 , thus the boundary lubricating regime was guaranteed for all tribotests. Each tribotest was performed at least three times to ensure the results reproductivity. The detailed experimental rig can be demonstrated in Figure 1.

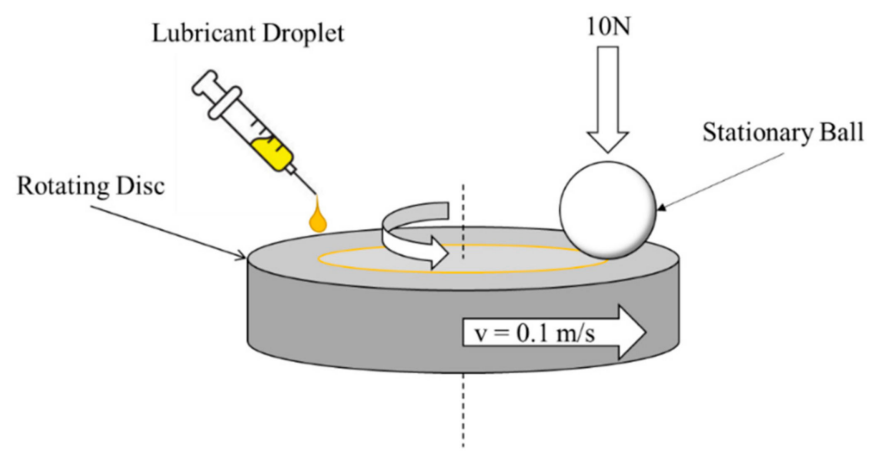

Figure 1. Schematic representing lubrication testing rig.

After the tests, the Contour GT-K 3D Optical Microscope (Billerica, MA, USA) was used to measure the depth and the width of the wear tracks. The wear loss volume of the 
ball was calculated based on the following formulas, where $d$ is the measured diameter of the wear scar and $r$ is the ball radius.

$$
\begin{gathered}
\text { Ball's volume loss }=\frac{\pi \mathrm{h}}{6} \times\left(\frac{3 \mathrm{~d}^{2}}{4}+\mathrm{h}^{2}\right) \\
\mathrm{h}=\mathrm{r}-\sqrt{\mathrm{r}^{2}-\frac{\mathrm{d}^{2}}{4}}
\end{gathered}
$$

\subsection{Characterization}

Scanning electron microscopy (SEM) (JEOL JSM-6490LV, Japan) coupled with energydispersive X-ray spectroscopy (EDS) was employed to morphologically and chemically analyze the obtained wear track morphology. Under high vacuum conditions, a $15-\mathrm{keV}$ accelerating voltage with a 10-mm working distance was applied to extract the morphology of the wear track and determine the elemental distribution. After that, a RENISHAW confocal Raman microscope (Woltton-under-Edge, UK) with a 532-nm laser line was applied to obtain the structural properties of the generated carbon-based tribofilm in terms of the $G$ band and D band.

\section{Results}

The disc wear track profile of the PAO oil shown in Figure 2a showed the most obvious wear scar with no additive. There was only a small variation in the disc's average surface roughness after the friction test rather than the distinct wear valleys, thus indicating that the disc wear loss was minimal. It can be seen that the non-sliding area exhibited the surface roughness of $R_{a}=0.574 \mu \mathrm{m}$, while the sliding area (disc wear track) showed $R_{a}=0.365 \mu \mathrm{m}$. On the other hand, the ball experienced a distinct wear loss that resulted in the circular wear scar with a surface roughness of $R_{a}=0.588 \mu \mathrm{m}$ (Figure $2 b$ ). Although the ball and disc were made of the same steel material, this phenomenon can be explained by the significantly higher original roughness of the discs $(0.578 \pm 0.088 \mu \mathrm{m})$ compared to the testing balls $(0.02 \mu \mathrm{m})$. In addition, the ball was continuously in contact and worn-out during sliding which resulted in a higher sliding distance in the ball compared to the disc (Figure 1). Therefore, under the boundary lubrication regime in the present test, there was only a small removal of the asperity peaks from the disc wear track which resulted in only micro-roughness change. Furthermore, the change in disc surface roughness became negligible when the lubricant additives were added to the base oil (Figures S1-S5) owing to their film formations along with the outstanding anti-wear performances which will be discussed later. As a result, this paper will report the wear loss of the ball and the change of the disc surface roughness.

\subsection{Frictional Test Results}

\subsubsection{Friction and Wear of PAO Oil with a Single Additive}

Figure 3 shows the friction and wear performances of the lubricants formulated with a single additive in PAO. According to Figure $3 \mathrm{a}$, once ZDDP was added to the PAO oil, a stable friction curve could be observed with a marked reduction from 0.14 to 0.108 . Meanwhile, the average steady-state friction of CPCa after 15 min-sliding was approximately 0.086 . This is also the lowest average coefficient of friction (COF) among all the pure additives tribotests, followed by Ni nanoparticles with approximately 0.103 (Figure 3b). However, the friction curve of CPCa exhibited a sharp upward trend followed by a moderate decline. 

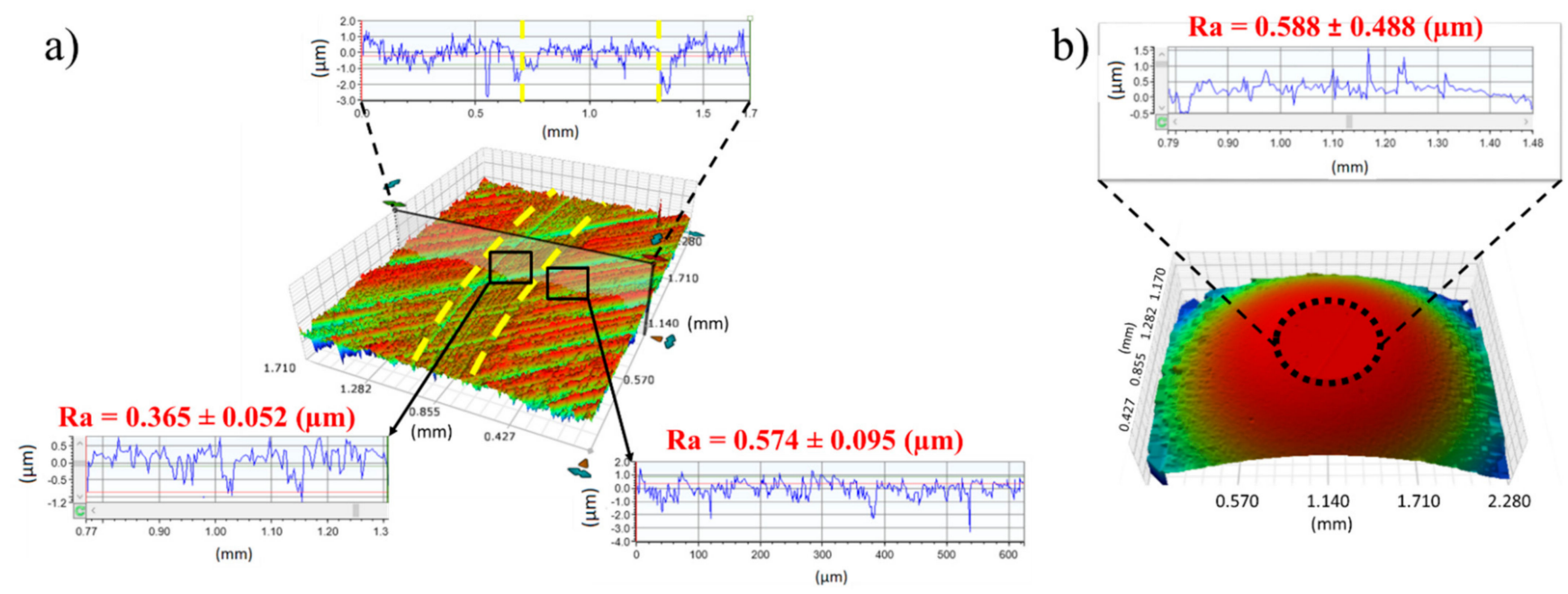

Figure 2. 3D image and scanning profile of (a) the disc and (b) the ball wear track of pure PAO oil tribotest.

a)

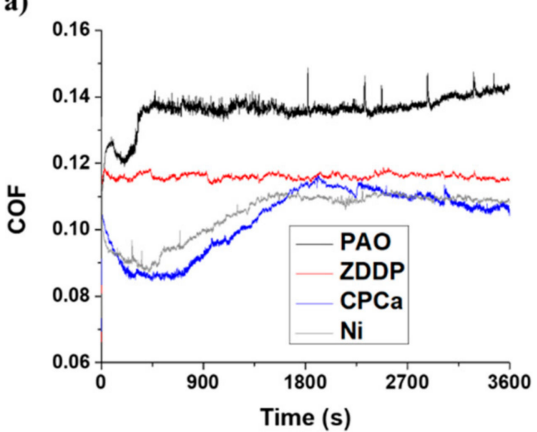

c)

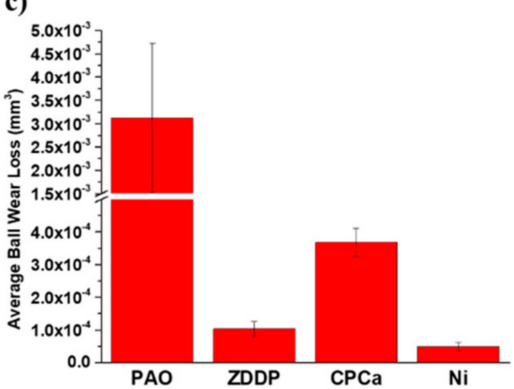

b)

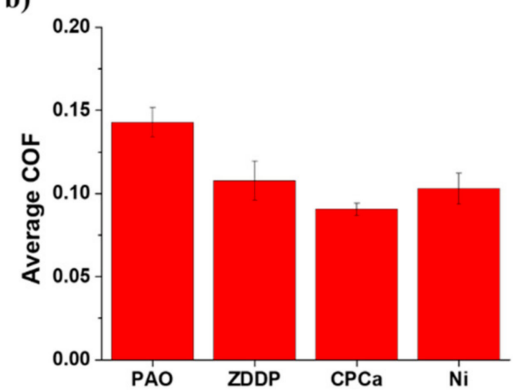

d)

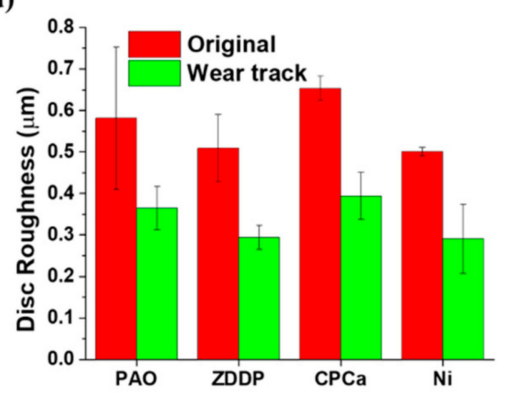

Figure 3. Tribological performance of zinc dialkyldithiophosphate (ZDDP), cyclopropanecarboxylic acid (CPCa), and Ni nanoparticles in Polyalphaolefin (PAO) oil: (a) Dynamic frictional curve; (b) Statistical average coefficient of friction (COF) at the steady-state; (c) Average ball wear loss; and (d) Variation of the surface roughness before and after sliding on the disc.

This behavior might be attributed to the weak adherence of the resulting carbonbased tribofilm on the sliding surfaces [13]. During sliding, the formation of carbon-based tribofilm was accompanied by the dissociation and polymerization of the CPCa molecules that deposit the thin carbon-based tribolayer on the sliding surfaces [14]. This film has bonding configurations similar to the diamond-like-carbon (DLC) which could reduce friction and wear. However, the bonding strength between such carbon-based film and steel surfaces is not sufficiently strong to accommodate the large plastic strain from the stressed-shearing effect [32]. As a consequence, the delamination of the carbon-based film occurred which increased friction followed by a slight decrease in friction due to the formation of the new carbon-based film from the surrounding CPCa molecules.

Meanwhile, despite the higher steady-state friction, the friction curve produced by solid Ni nanoparticles had a much more stable characteristic than the CPCa after 25 min 
of sliding. The initial fluctuation of Ni nanoparticles' friction curve was attributed to the rolling effect of these particles which could separate direct contacts between asperities and lowered the friction [33]. Subsequently, the agglomeration of the nanoparticles occurred during sliding that resulted in a gradual increase in friction coefficient [28]. These aggregated clusters of nanoparticles were then deformed and compacted into a protective film on the sliding surfaces that stabilized the friction curve [25,34].

From Figure $3 c$, the non-additive tribotests caused the average $31.3 \times 10^{-4} \mathrm{~mm}^{3}$ average ball wear loss. Nevertheless, the addition of the additives dramatically improved the wear loss. Among all experimental additives, CPCa resulted in the highest ball wear loss volumes $\left(3.7 \times 10^{-4} \mathrm{~mm}^{3}\right)$ which is 2.6 times higher than ZDDP (approximately $1.03 \times 10^{-4} \mathrm{~mm}^{3}$ ). In contrast, Ni nanoparticles exhibited an outstanding anti-wear performance in PAO oil. Its ball wear loss of $4.89 \times 10^{-5} \mathrm{~mm}^{3}$ was $52.5 \%$ lower than the ZDDP test. Based on the disc wear track roughness in Figure 3d, Ni nanoparticles produced a higher variation roughness compared to other tribotests. This may be due to the uneven distribution of solid nanoparticles and agglomerated clusters across the interface during sliding. Meanwhile, CPCa produced a slightly higher average disc wear track roughness compared to other additives which might be attributed to the uneven deposition of the carbon debris from the delamination of the carbon-based tribofilm across the sliding area.

\subsubsection{Friction and Wear of PAO Oil with Mixed Additives}

The tribological outcomes of ZDDP, ZDDP+CPCa, and ZDDP+Ni are illustrated in Figure 4. It is clear from Figure $4 \mathrm{a}$ that there was a small impact in friction performance of ZDDP when Ni nanoparticles were further added. However, despite the initial sharp decrease, a dramatic upward frictional trend could be clearly observed when CPCa was blended with ZDDP. It can be concluded that the friction performance of each additive has deteriorated. Based on Figure $4 b$, the average COF of ZDDP+CPCa lubricant was 0.119. Meanwhile, the presence of Nickel nanoparticles in PAO oil containing ZDDP resulted in the average friction of 0.114 with a lower standard deviation compared to the average value of 0.108 from ZDDP alone tests.

a)

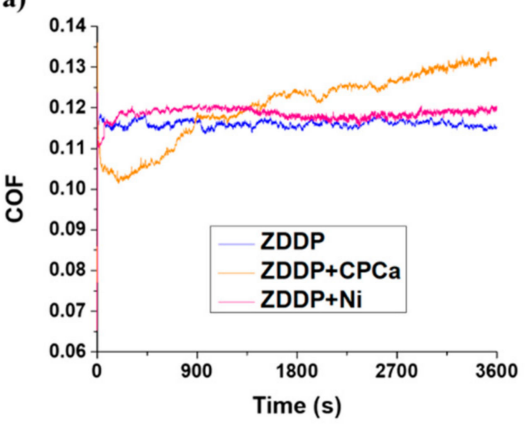

c)

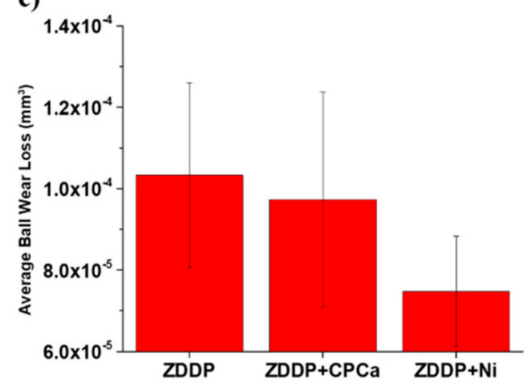

b)

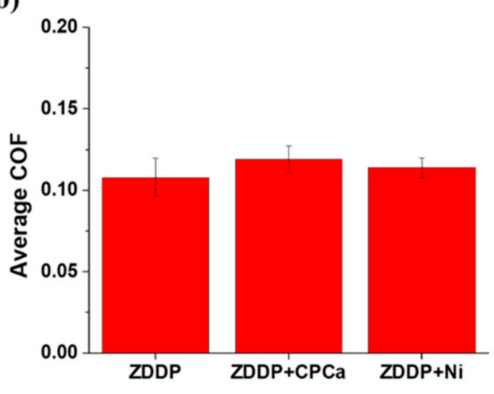

d)

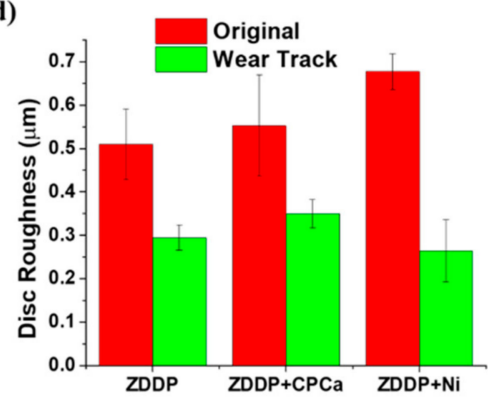

Figure 4. Tribological performance of ZDDP, CPCa+ZDDP, and ZDDP+Ni nanoparticles in PAO oil: (a) dynamic frictional curve; (b) statistical average COF at the steady-state; (c) average ball wear loss; and (d) variation of the surface roughness before and after sliding on the disc. 
On the other hand, the combination of Ni nanoparticles and ZDDP produced $27.6 \%$ lower ball wear loss compared to ZDDP alone (Figure 4c). Interestingly, despite the higher original disc roughness compare to other testing discs, Ni nanoparticles lowered slightly the roughness of ZDDP (Figure 4d). The high standard deviation of this wear track roughness may be due to the uneven distribution of residual nanoparticles across the wear track. Meanwhile, it can be hypothesized that there was an incompatibility between the ZDDP and CPCa which resulted in a rougher wear track compare to the PAO oil with added ZDDP alone. Finally, although the combination of CPCa and ZDDP produced slightly lower ball wear loss, it is noteworthy that the variation among the experimental outcomes of this tribotest was high which indicated an unstable anti-wear performance and the difficulty in achieving repeatable outcomes (Figure 4c).

\subsubsection{Summary of Tribological Results}

Shown in Table 2 is the summary of the friction and wear results of the tested lubricants. It can be seen that the ball wear loss was significantly reduced under the lubrication of Ni nanoparticles alone while CPCa performed best in friction reduction. However, it is noticeable that the tribotests containing this carbon precursor additive resulted in the highest ball and disc wear surfaces' roughness besides the no additive PAO oil. This may be the consequence of the distinct increase in friction (Figures 3a and 4a) and results in the higher ball wear loss volume compared to other tested additives. Compared to the previous study by Johnson et al. [13], these results were slightly different because of the longer testing duration. In contrast, the wear surface roughness of ball and disc from $\mathrm{ZDDP}, \mathrm{Ni}$, and $\mathrm{ZDDP}+\mathrm{Ni}$ tribotests were relatively lower than other experiments. This indicates a more stable tribofilm formation rate as evidenced by the stable friction curve and exhibited better anti-wear performance than the tribotests containing CPCa. To verify these hypotheses, further analyses with chemical characterization on the ball wear scar were conducted.

Table 2. Summary of tribological results.

\begin{tabular}{|c|c|c|c|c|}
\hline $\begin{array}{l}\text { Experimental } \\
\text { Sample }\end{array}$ & $\begin{array}{c}\text { Steady-State } \\
\text { Friction }\end{array}$ & $\begin{array}{c}\text { Disc Wear Surface } \\
\text { Roughness } \\
(\mu \mathrm{m})\end{array}$ & $\begin{array}{c}\text { Ball Wear } \\
\text { Volume } \\
\left(10^{-4} \mathrm{~mm}^{3}\right)\end{array}$ & $\begin{array}{c}\text { Ball Wear Surface } \\
\text { Roughness } \\
(\mu \mathrm{m})\end{array}$ \\
\hline $\mathrm{PAO}$ & $0.143 \pm 0.009$ & $0.365 \pm 0.052$ & $3.130 \pm 1.590$ & $0.588 \pm 0.488$ \\
\hline ZDDP & $0.108 \pm 0.011$ & $0.295 \pm 0.029$ & $0.103 \pm 0.023$ & $0.130 \pm 0.068$ \\
\hline $\mathrm{CPCa}$ & $0.090 \pm 0.004$ & $0.395 \pm 0.057$ & $0.368 \pm 0.043$ & $0.170 \pm 0.011$ \\
\hline $\mathrm{Ni}$ & $0.103 \pm 0.009$ & $0.291 \pm 0.083$ & $0.049 \pm 0.012$ & $0.140 \pm 0.031$ \\
\hline $\mathrm{ZDDP}+\mathrm{Ni}$ & $0.114 \pm 0.006$ & $0.264 \pm 0.072$ & $0.075 \pm 0.014$ & $0.148 \pm 0.039$ \\
\hline $\mathrm{ZDDP}+\mathrm{CPCa}$ & $0.119 \pm 0.008$ & $0.350 \pm 0.032$ & $0.097 \pm 0.026$ & $0.205 \pm 0.067$ \\
\hline
\end{tabular}

\subsection{Wear Track Morphology of Ball Counterpart}

Figure 5 highlights the ball wear track morphology of the tested lubricants. It can be observed that ZDDP remarkably improved the wear-resistance of the ball when added to PAO oil. The oil base containing this conventional additive produced a $274 \mu \mathrm{m}$ diameter circular wear scar (Figure 5b) which was 54\% smaller than the $600 \mu \mathrm{m}$ diameter of the pure oil (Figure 5a). There was an appearance of the dark cluster that can be detected on the ridge area of the wear scar. They might perform as a cushion to prevent the direct asperity contact between two substrates which results in a narrower valley in the middle of the wear scar morphology and a significantly smaller scar roughness $\left(R_{a}=0.130 \mu \mathrm{m}\right)$ compared to the PAO oil test $\left(\mathrm{R}_{\mathrm{a}}=0.588 \mu \mathrm{m}\right)$. Meanwhile, despite the slightly lower wear diameter (about $400 \mu \mathrm{m}$ ), the wear morphology in CPCa tribotest (Figure 5c) was similar to the pure PAO oil with the presence of various deep grooves and smearing across the interfacing areas. When the concentration of ZDDP was reduced by half when combined 
with CPCa (Figure 5d), a lighter film could be observed, discretely across the center of the ball's interfacing area instead of significantly concentrating on localized areas like ZDDP alone. It was also the roughest ball wear scar $\left(R_{a}=0.205 \mu \mathrm{m}\right)$ after the non-additive oil tribotests. In contrast, Ni nanoparticles emerged as the most outstanding anti-wear additive with the lowest wear loss. Besides ZDDP alone, the tribotests containing this additive resulted in the lowest ball wear scars' roughness among all experimental lubricants (Table 2). With pure PAO oil, black clusters could be detected in the boundary areas and the deep grooves of wear morphology (Figure 5e). When the Ni nanoparticles were added to PAO oil containing ZDDP, the wear scar diameter decreased from $274 \mu \mathrm{m}$ to $254 \mu \mathrm{m}$. Additionally, a unique brown color film could be observed across the relative smooth wear morphology. It is noticeable that there were fewer abrasive grooves from the ZDDP+Ni tribotest compared to the Ni nanoparticle alone. This might be attributed to the synergistic effect between ZDDP film and Ni nanoparticles' protective film.

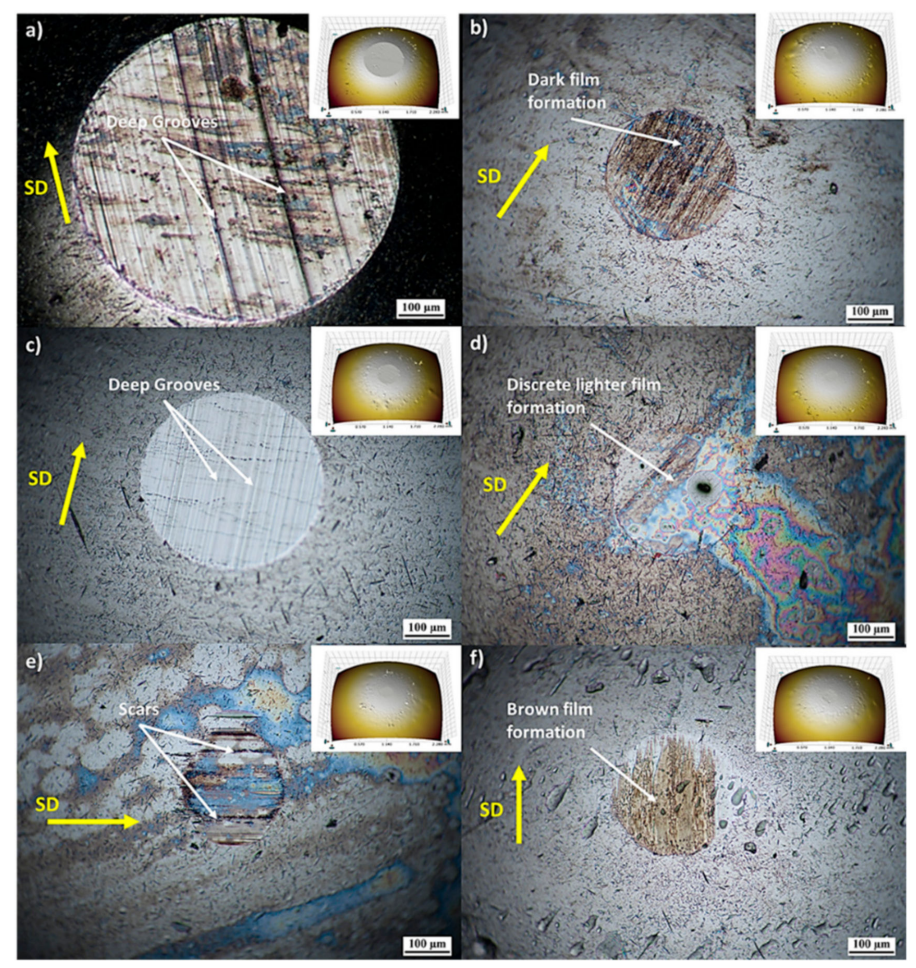

Figure 5. Ball wear morphology of (a) pure PAO oil; (b) ZDDP; (c) CPCa; (d) ZDDP+CPCa; (e) Ni; and (f) ZDDP+Ni tribotest.

\subsection{Compositional Analysis of the Ball Wear Surfaces}

\subsubsection{PAO Oil Tribotests with a Single Additive}

Figure 6 illustrates the SEM and EDS results from the lubricants of PAO oil containing a single additive. The EDS signal of carbon (C) indicated the formation of black carbon clusters that were found on the wear tracks of CPCa, ZDDP, and PAO tribotests. The $\mathrm{C}$-signal in PAO oil containing ZDDP might be attributed to the oil dissociation, while the detected C-signal of the CPCa test could be due to the in situ formation of carbonbased tribofilm [13]. In addition, various micro-streaks appeared on the SEM image of CPCa wear track (Figure 6b) which indicated a soft film formation that filled the valleys [13]. In contrast, the solid debris, which played as the third-body abrasive particles, was formed on the pure oil case as shown by numerous abrasive scars on the ball's wear area (Figure 6a). Meanwhile, the ZDDP tribofilm formed on the contacting area consisted of distinct signals of zinc, sulphur, and phosphorus elements by EDS mapping (Figure 6c). 

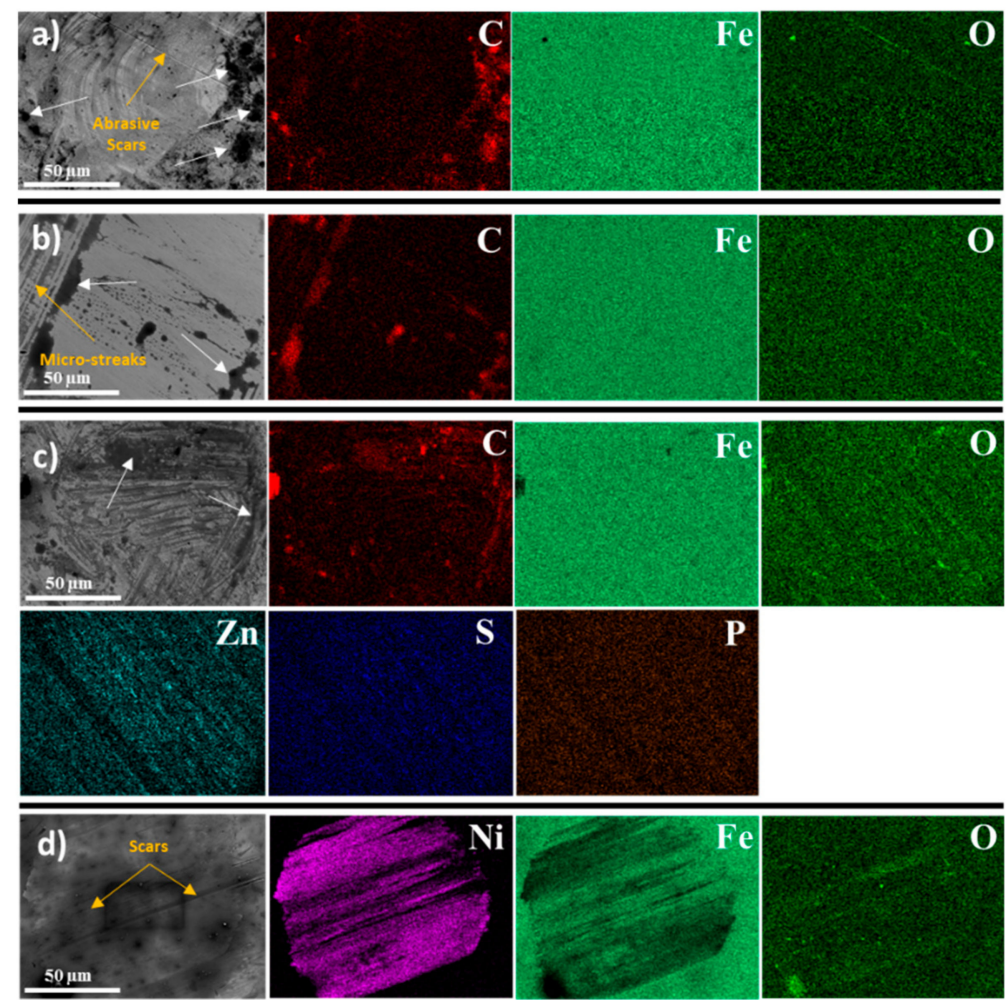

Figure 6. SEM and EDS outcomes of (a) PAO, (b) CPCa, (c) ZDDP, and (d) Ni nanoparticles (where the white arrow indicates the location of detected carbon cluster).

On the other hand, the most interesting aspect was the compact Ni-rich tribofilm that could be clearly observed on the sliding surface (Figure $6 \mathrm{~d}$ ). This proved the mending effect of these nanoparticles. Due to the soft characteristic (about 4.0 Mohr hardness) along with the nano-dimensions, these solid particles penetrated to the surface roughness to promote a compact protective layer. In particular, the Ni nanoparticles experienced compression and deformation under a stressed-shearing effect at the sliding interfaces that in turn was welded on the counterpart's surfaces due to flash temperature [25]. The protective film, thus, covered significantly the interfacing ball area and weaken the Fe signal. This Ni-rich layer not only improved remarkably the wear-resistance of the siding ball (Figure 3c) but also enhanced the load-carrying capacity and resulted in a stable friction curve at the steady-state (Figure 3a) [25]. Additionally, narrow scars on this wear scar surface indicate the occurrence of either nanoparticle agglomeration [28] or the delamination of the soft resultant film. The even distribution of the oxygen $(\mathrm{O})$ signal across the wear track indicates the formation of $\mathrm{NiO}$ along with the iron oxide under high pressure.

\subsubsection{PAO Oil Tribotests with Mixed Additives}

It is clear from Figure 7 that the ZDDP tribofilm formed in all lubricants with two additives showed the detected signals of zinc, sulphur, and phosphorus elements on the contacting area. From Figure 7a of ZDDP sliding tests, a rough surface was produced on the ball sliding area. As mentioned above, the black carbon-rich clusters were also formed on these tribotests. However, when CPCa, as a carbon-precursor additive, was added (ZDDP+CPCa tribotest), the carbon-signal was significantly weakened (Figure $7 \mathrm{~b}$ ). This can be explained by the large removal of carbon-based tribofilm in this formulation. The obtained EDS signal of zinc and sulphur in this sample was also slightly weaker than ZDDP tribotests due to a lower concentration of the conventional additive. However, instead of evenly distributed across the wear track, the ZDDP-rich film, in this case, formed in certain areas of the wear scar. This supported the hypothesis that ZDDP and CPCa functioned separately by forming the tribofilms in separated positions of the sliding area. 
Under boundary lubrication, the carbon-based film could be continuously removed while the ZDDP film formed discretely and became compacted on the scar surface. This not only resulted in a non-uniform wear surface but also destabilized the anti-wear property of PAO containing ZDDP lubricant with a possibility of increasing friction (Figure 4a). In contrast, the tribological performance of the ZDDP and Ni combination was significantly better than ZDDP by itself. According to Figure 7c, the synergistic effect between Ni nanoparticle's film and ZDDP film could be observed which was evidenced by the compact EDS signal of zinc and sulphur at the location where Ni-rich film was formed. Nevertheless, the iron-signal at this location was slightly weaker compared to other areas of this iron-EDS mapping and the tribofilm of ZDDP individually (Figure 7a). Moreover, many scratches can be found on the wear track. These may be attributed to the soft characteristic and the delamination of nickel-film during the tribotests.

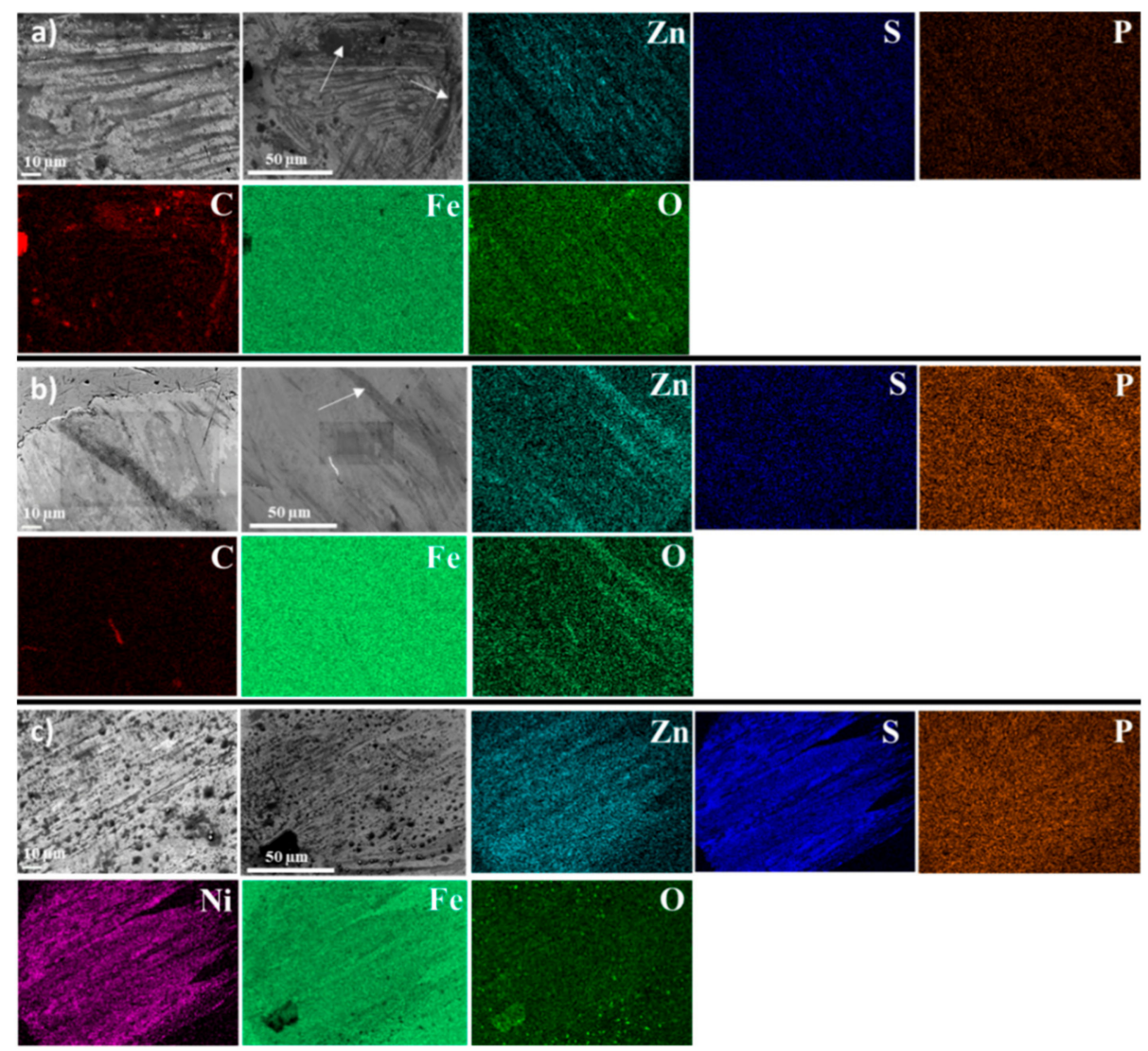

Figure 7. SEM and EDS outcomes of (a) ZDDP, (b) ZDDP+CPCa, and (c) ZDDP+Ni tribotests. (The white arrow indicates the location of detected rich ZDDP film formation).

\section{Discussion}

\subsection{ZDDP and CPCa Combination}

To analyze the structural characteristics of obtained carbon-based tribofilm in the wear scar, Raman analysis was carried out to detect the D band and G band of carbon bonding configurations. It was clear from the deconvolution outcomes in Figure 8 that there was no detected signal from PAO and ZDDP. This indicated that the carbon cluster from the EDS signal in pure PAO oil tests and PAO oil containing ZDDP tests were attributed to the oil dissociation that might produce the carbon soot particles. In contrast, distinct peaks representing the high intensity of the $\mathrm{D}$ band and $\mathrm{G}$ band could be observed from the lubricant containing CPCa by itself. However, the intensity of these peaks decreased dramatically in the ZDDP+CPCa tribotest which can be explained by the reduction of carbon-precursor additive concentration and the competitive effect between these added additives. In addition, the $\mathrm{I}_{\mathrm{D}} / \mathrm{I}_{\mathrm{G}}$ ratio of 0.786 for this combination was slightly higher than 
the ratio of $0.764 \mathrm{CPCa}$ with pure $\mathrm{PAO}$ oil. This indicates a negligible variation in graphitic feature of carbon-based tribofilm forming from ZDDP+CPCa and CPCa only tribotests. As a result, it can be concluded that the CPCa additive in two experiments resulted in a carbonfilm formation with a significantly high graphitic degree that could deliver remarkable anti-friction property [35]. However, this carbon-based tribofilm also exhibited a soft characteristic [12] with low absorption strength to the steel substrate as evidenced by the distinct increase of friction curve after a certain sliding duration (Figures 3a and 4a). Additionally, the formation of oxide debris which caused noisy Raman signals provided evidence to support these claims [36]. The Raman signal of carbon-based film from CPCa in combination with ZDDP contained much fewer noise signals, which was attributed to the functional property of ZDDP as an antioxidant [3] to inhibit the development of oxide scale following by the formation of oxide debris. Together with the EDS results (Figure $7 \mathrm{~b}$ ), it can be concluded that the combination of CPCa and ZDDP resulted in an alternative formation of in situ carbon-based film and ZDDP film on the steel substrate, instead of the hierarchical structure of ZDDP film overlapping the carbon-based film [37]. The wear surface, in this case, was not homogeneous because of the alternative formation of soft carbon-based films along with the discrete ZDDP film. While the carbon-based tribofilm was removed quickly, the discrete ZDDP film survived and remained on the wear track. This is the main reason why this combination exhibited a less stable anti-wear performance compared to ZDDP alone. Therefore, to reduce the added amount of ZDDP by the carbon-precursor additive, further research should be focused on improving the absorption strength of carbon-based tribofilm formation.

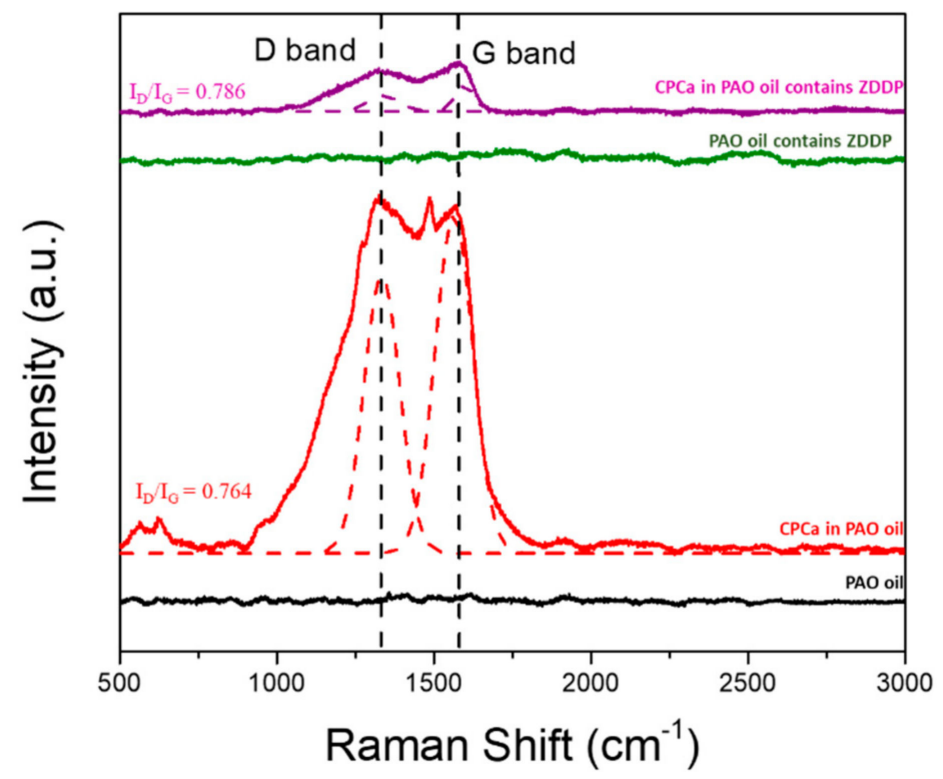

Figure 8. Raman analysis of the ball wear surfaces after lubricated by PAO oil, CPCa in PAO oil, ZDDP in PAO oil, and CPCa+ZDDP in PAO oil.

\subsection{ZDDP and Ni Nanoparticles Combination}

Based on the wear outcomes (Figure 4c) as well as SEM and EDS analysis (Figure 7c), a synergistic effect between the ZDDP film with the Ni nanoparticles' protective film was indicated. This could be demonstrated further by the EDS intensity of the sulphur element (Figure 9) which showed a more effective sulphur-rich layer as the foundation layer of ZDDP-derived film formation when Ni nanoparticles were further added. Besides the iron cation, it was shown by Spikes et al. [3] that the ZDDPs' dithiophosphate ligands can also be exchanged from $\mathrm{Zn}^{2+}$ to $\mathrm{Ni}^{2+}$ which may be activated via triboemission [8] evidenced by the formation of $\mathrm{NiO}$ across the $\mathrm{ZDDP}+\mathrm{Ni}$ experiment (Figure 7c). Thus, nickel dithiophosphate as new compounds of dithiophosphate ligands with less thermal stability was formed. Under extreme pressure and flash temperature activation, it was 
hypothesized that these dithiophosphate compounds will decompose and create NiS, along with $\mathrm{ZnS}$ and FeS in the sulfide layer of resultant tribofilm [38]. Recent studies on the forming mechanism of ZDDP film by Shimizu et al. on rotation motion [39] and reciprocating motion [40] highlighted the important role of this sulfide-rich layer in the formation as well as the tribological characteristic of this tribofilm. In particular, at the beginning of the ZDDP sliding experiment, a sulphur-rich film is spontaneously formed in response to the extreme pressure condition before the phosphorus-rich film, having a slower formation rate, is promoted. This sulphur-rich film improves the anti-wear property of the oil-based lubricant. However, under boundary conditions, this sulphur-rich film is usually removed quickly while the phosphorus-film is not promoted which results in a severely worn area with enormous scars and solids particles [39]. In this study, thanks to the appearance of nickel nanoparticles across the contacting area, the absorption strength of the sulphur-rich film has been improved before the hierarchical structure of the tribofilm was fully developed, as shown by the compact wear track surface and the lower wear loss volume compared to ZDDP alone (Figure 4c). In addition, the NiS also has a lower hardness (around 3.0-3.5 Mohr hardness) compared to ZnS (about 3.8 Mohr hardness). Thus, the appearance of NiS in this film might "soften" the sliding area which improves the anti-wear performance of ZDDP additive in PAO oil. From this result, a future study utilizing the synergy between nanomaterials' protective film and ZDDP film to reduce the usage amount of ZDDP is highly recommended.

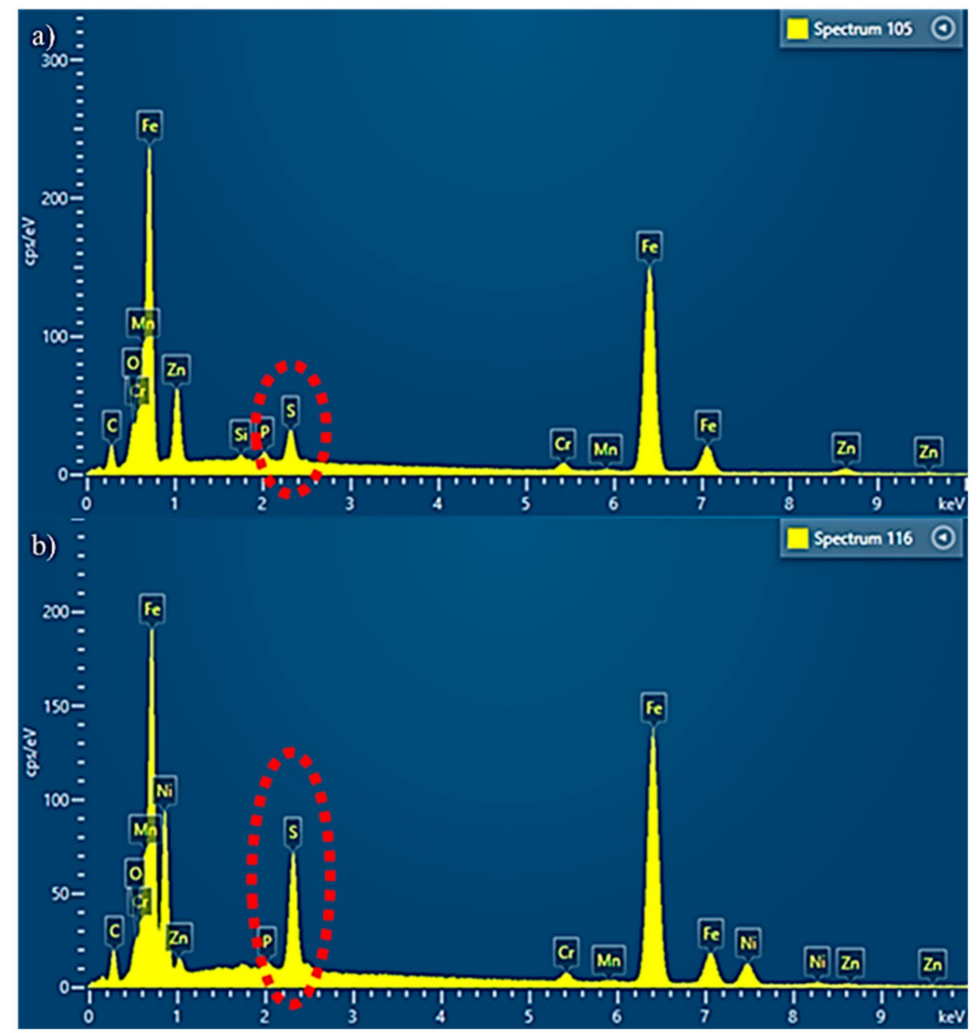

Figure 9. EDS intensity from the obtained ball wear tracks lubricated by ZDDP in (a) PAO oil and (b) ZDDP+Ni in PAO oil.

\section{Conclusions}

In summary, this study has investigated the influence of CPCa and Ni nanoparticles on the tribological performance of ZDDP in PAO oil ambient. In particular, a synergistic effect between ZDDP and Ni nanoparticles was evidenced which improved further the anti-wear performance of ZDDP (by $27.6 \%$ ). This was due to the promotion of sulfide layer formation with an aid of Ni nanoparticles that resulted in the compacted Ni-S tribofilm on the sliding surfaces. In contrast, $\mathrm{CPCa}$, as a carbon-based tribofilm precursor, exhibited 
a competitive effect against the ZDDP film formation. It was closely associated with the non-compatibility and low affinity between the soft carbon-based and ZDDP tribofilms which resulted in the discontinuous ZDDP tribofilm formation on the wear surfaces. The finding in this study has further advanced the understanding of the tribochemistry between ZDDP and other additives which is beneficial to improving the tribological performance of engine oil in the cold start-up's process with limited use of ZDDP.

Supplementary Materials: The following are available online at https:/ / www.mdpi.com/article/10 $.3390 /$ lubricants $9040035 /$ s1, Detail profile of experimental disc roughness of the additive tribotests and their wear morphologies coupling with the corresponding balls' wear surface roughness can be found in the Supporting Information (Figures S1-S5).

Author Contributions: K.K.H. prepared the samples, conducted the tribotests, did the friction wear and calculation, wrote the manuscript, and conceptualized, investigated, and cured the data. K.A.T. provided supervision, writing - review and editing, and funding acquisition. S.T.P. provided support in writing-review and editing, SEM-EDS characterization, and Raman acquisitions. All authors have read and agreed to the published version of the manuscript.

Funding: The study is funded by Australian Research Council (ARC) Discovery Project DP190103455.

Institutional Review Board Statement: Not applicable.

Informed Consent Statement: Not applicable.

Data Availability Statement: Data only available on request due to the protection of IP.

Acknowledgments: The authors acknowledge the use of the JEOL 6490 SEM at the UOW Electron Microscopy Centre. The authors thank the Australian National Fabrication Facility (ANFF)-Materials Node for the use of Raman facilities.

Conflicts of Interest: The authors declare no conflict of interest.

\section{References}

1. Macian, V.; Tormos, B.; Ruiz, S.; Miró, G. Low viscosity engine oils: Study of wear effects and oil key parameters in a heavy duty engine fleet test. Tribol. Int. 2016, 94, 240-248. [CrossRef]

2. Ueda, M.; Kadiric, A.; Spikes, H. On the crystallinity and durability of ZDDP tribofilm. Tribol. Lett. 2019, 67, 123. [CrossRef]

3. Spikes, H. The history and mechanisms of ZDDP. Tribol. Lett. 2004, 17, 469-489. [CrossRef]

4. Martin, J.M. Antiwear mechanisms of zinc dithiophosphate: A chemical hardness approach. Tribol. Lett. 1999, 6, 1-8. [CrossRef]

5. Nicholls, M.A.; Do, T.; Norton, P.R.; Kasrai, M.; Bancroft, G.M. Review of the lubrication of metallic surfaces by zinc dialkyldithiophosphates. Tribol. Int. 2005, 38, 15-39. [CrossRef]

6. Spikes, H. Low- and zero-sulphated ash, phosphorus and sulphur anti-wear additives for engine oils. Lubr. Sci. 2008, 20, 103-136. [CrossRef]

7. Dawczyk, J.; Morgan, N.; Russo, J.; Spikes, H. Film thickness and friction of ZDDP tribofilms. Tribol. Lett. 2019, 67, 34. [CrossRef]

8. Zhang, J.; Spikes, H. On the mechanism of ZDDP antiwear film formation. Tribol. Lett. 2016, 63, 24. [CrossRef]

9. Elo, R.; Jacobson, S. Formation and breakdown of oil residue tribofilms protecting the valves of diesel engines. Wear 2015, 330, 193-198. [CrossRef]

10. Wong, V.W.; Tung, S.C. Overview of automotive engine friction and reduction trends-Effects of surface, material, and lubricantadditive technologies. Friction 2016, 4, 1-28. [CrossRef]

11. Chen, X.; Li, J. Superlubricity of carbon nanostructures. Carbon 2020, 158, 1-23. [CrossRef]

12. Erdemir, A.; Donnet, C. Tribology of diamond-like carbon films: Recent progress and future prospects. J. Phys. Appl. Phys. 2006, 39, R311. [CrossRef]

13. Johnson, B.; Wu, H.; Desanker, M.; Pickens, D.; Chung, Y.-W.; Wang, Q.J. Direct formation of lubricious and wear-protective carbon films from phosphorus-and sulfur-free oil-soluble additives. Tribol. Lett. 2018, 66, 2. [CrossRef]

14. Wu, H.; Khan, A.M.; Johnson, B.; Sasikumar, K.; Chung, Y.-W.; Wang, Q.J. Formation and Nature of Carbon-Containing Tribofilms. ACS Appl. Mater. Interfaces. 2019, 11, 16139-16146. [CrossRef] [PubMed]

15. Khan, A.M.; Wu, H.; Ma, Q.; Chung, Y.-W.; Wang, Q.J. Relating Tribological Performance and Tribofilm Formation to the Adsorption Strength of Surface-Active Precursors. Tribol. Lett. 2020, 68, 6. [CrossRef]

16. Haque, T.; Morina, A.; Neville, A.; Kapadia, R.; Arrawsmith, S. Study of the ZDDP antiwear tribofilm formed on the DLC coating using AFM and XPS techniques. In Automotive Lubricant Testing and Advanced Additive Development; ASTM International: New York, NY, USA, 2008.

17. Topolovec-Miklozic, K.; Lockwood, F.; Spikes, H. Behaviour of boundary lubricating additives on DLC coatings. Wear 2008, 265, 1893-1901. [CrossRef] 
18. Dai, W.; Kheireddin, B.; Gao, H.; Liang, H. Roles of nanoparticles in oil lubrication. Tribol. Int. 2016, 102, 88-98. [CrossRef]

19. Lee, K.; Hwang, Y.; Cheong, S.; Choi, Y.; Kwon, L.; Lee, J.; Kim, S.H. Understanding the role of nanoparticles in nano-oil lubrication. Tribol. Lett. 2009, 35, 127-131. [CrossRef]

20. Spikes, H. Friction modifier additives. Tribol. Lett. 2015, 60, 5. [CrossRef]

21. Zhang, S.; Hu, L.; Feng, D.; Wang, H. Anti-wear and friction-reduction mechanism of Sn and Fe nanoparticles as additives of multialkylated cyclopentanes under vacuum condition. Vacuum 2013, 87, 75-80. [CrossRef]

22. Padgurskas, J.; Rukuiza, R.; Prosyčevas, I.; Kreivaitis, R. Tribological properties of lubricant additives of Fe, Cu and Co nanoparticles. Tribol. Int. 2013, 60, 224-232. [CrossRef]

23. Kotnarowski, A. Tribological properties of oils modified with the addition of metal nanoparticles. Solid State Phenom. 2006, 113, 393-398. [CrossRef]

24. Abad, M.; Sánchez-López, J. Tribological properties of surface-modified Pd nanoparticles for electrical contacts. Wear 2013, 297, 943-951. [CrossRef]

25. Chou, R.; Battez, A.H.; Cabello, J.; Viesca, J.; Osorio, A.; Sagastume, A. Tribological behavior of polyalphaolefin with the addition of nickel nanoparticles. Tribol. Int. 2010, 43, 2327-2332. [CrossRef]

26. Pham, S.T.; Tieu, A.K.; Sencadas, V.; Lei, W.; Liu, D.; Wan, S.; Hao, J. Smart-Responsive Colloidal Capsules as an Emerging Tool to Design a Multifunctional Lubricant Additive. ACS Appl. Mater. Interfaces. 2021, 13, 7714-7724. [CrossRef]

27. Pham, S.T.; Tieu, A.K.; Wan, S.; Hao, J.; Zhu, H.; Nguyen, H.H.; Mitchell, D.R. Oxidative and Frictional Behavior of a Binary Sodium Borate-Silicate Composite in High-Temperature Lubricant Applications. Ind. Eng. Chem. Res. 2019, 59, $2921-2933$. [CrossRef]

28. Pham, S.T.; Wan, S.; Tieu, K.A.; Ma, M.; Zhu, H.; Nguyen, H.H.; Mitchell, D.R.; Nancarrow, M.J. Unusual Competitive and Synergistic Effects of Graphite Nanoplates in Engine Oil on the Tribofilm Formation. Adv. Mater. Interfaces 2019, 6, 1901081. [CrossRef]

29. Matsui, Y.; Aoki, S.; Masuko, M. Influence of coexisting functionalized polyalkylmethacrylates on the formation of ZnDTP-derived tribofilm. Tribol. Int. 2016, 100, 152-161. [CrossRef]

30. Roberts, A.; Brooks, R.; Shipway, P. Internal combustion engine cold-start efficiency: A review of the problem, causes and potential solutions. Energy Convers. Manag. 2014, 82, 327-350. [CrossRef]

31. Hamrock, B.J.; Dowson, D. Isothermal elastohydrodynamic lubrication of point contacts: Part III-fully flooded results. J. Lubr. Tech. 1977, 99, 264-275. [CrossRef]

32. Mori, H.; Tachikawa, H. Increased adhesion of diamond-like carbon-Si coatings and its tribological properties. Surface Coat. Technol. 2002, 149, 224-229. [CrossRef]

33. Wu, Y.; Tsui, W.; Liu, T. Experimental analysis of tribological properties of lubricating oils with nanoparticle additives. Wear 2007, 262, 819-825. [CrossRef]

34. Hu, J.; Zhang, Y.; Yang, G.; Gao, C.; Song, N.; Zhang, S.; Zhang, P. In-situ formed carbon based composite tribo-film with ultra-high load bearing capacity. Tribol. Int. 2020, 152, 106577. [CrossRef]

35. Hoffman, E.E.; Marks, L.D. Graphitic carbon films across systems. Tribol. Lett. 2016, 63, 32. [CrossRef]

36. Soler, M.A.; Qu, F. Raman spectroscopy of iron oxide nanoparticles. In Raman Spectroscopy for Nanomaterials Characterization; Springer: Berlin/Heidelberg, Germany, 2012; pp. 379-416.

37. Vengudusamy, B.; Green, J.H.; Lamb, G.D.; Spikes, H.A. Durability of ZDDP tribofilms formed in DLC/DLC contacts. Tribol. Lett. 2013, 51, 469-478. [CrossRef]

38. Heuberger, R.; Rossi, A.; Spencer, N.D. XPS study of the influence of temperature on ZnDTP tribofilm composition. Tribol. Lett. 2007, 25, 185-196. [CrossRef]

39. Shimizu, Y.; Spikes, H.A. The influence of slide-roll ratio on ZDDP tribofilm formation. Tribol. Lett. 2016, 64, 19. [CrossRef]

40. Shimizu, Y.; Spikes, H.A. The tribofilm formation of ZDDP under reciprocating pure sliding conditions. Tribol. Lett. 2016, 64, 46. [CrossRef] 